\title{
Memetic electromagnetism algorithm for surface reconstruction with rational bivariate Bernstein basis functions
}

\author{
Andrés Iglesias $^{1,2}$ (1) Akemi Gálvez ${ }^{1,2}$
}

Published online: 9 June 2016

(C) Springer Science+Business Media Dordrecht 2016

\begin{abstract}
Surface reconstruction is a very important issue with outstanding applications in fields such as medical imaging (computer tomography, magnetic resonance), biomedical engineering (customized prosthesis and medical implants), computer-aided design and manufacturing (reverse engineering for the automotive, aerospace and shipbuilding industries), rapid prototyping (scale models of physical parts from CAD data), computer animation and film industry (motion capture, character modeling), archaeology (digital representation and storage of archaeological sites and assets), virtual/augmented reality, and many others. In this paper we address the surface reconstruction problem by using rational Bézier surfaces. This problem is by far more complex than the case for curves we solved in a previous paper. In addition, we deal with data points subjected to measurement noise and irregular sampling, replicating the usual conditions of real-world applications. Our method is based on a memetic approach combining a powerful metaheuristic method for global optimization (the electromagnetism algorithm) with a local search method. This method is applied to a benchmark of five illustrative examples exhibiting challenging features. Our experimental results show that the method performs very well, and it can recover the underlying shape of surfaces with very good accuracy.
\end{abstract}

Andrés Iglesias

iglesias@unican.es;

http://personales.unican.es/iglesias

1 Department of Applied Mathematics and Computational Sciences, E.T.S.I. Caminos, Canales y Puertos, University of Cantabria, Avda. de los Castros, s/n, 39005 Santander, Spain

2 Department of Information Science, Faculty of Science, Narashino Campus, Toho University, 2-2-1 Miyama, Funabashi 274-8510, Japan
Keywords Surface reconstruction - Rational surfaces · Memetic approach · Electromagnetism algorithm $\cdot$ Local search

\section{Introduction}

\subsection{Motivation}

The problem of obtaining a mathematical surface fitting a given set of data points (usually referred to as surface approximation or surface reconstruction) has been a very hot topic of research during the last few decades. From a theoretical point of view, it is a key tool in fields such as approximation theory (Cox 1993; Franke and Schumaker 1986), statistics (Draper and Smith 1998), numerical analysis (Forsey and Bartels 1995; Pottmann et al. 2002), geometric modeling (Eck and Hoppe 1996; Gálvez et al. 2007; Varady and Martin 2002) and computer-aided geometric design (CAGD) (Iglesias and Gálvez 2001; Piegl and Tiller 1997). Most of interest on this subject can be attributed to the wide range of applications of this technology in several fields. A well-known example is given by reverse engineering, a field where a (usually large) collection of data points is acquired from an already existing physical object. These data points are then approximated by mathematical functions in order to obtain a fully usable digital model (Barhak and Fischer 2001; Hoffmann 2005; Ma and Kruth 1995). There are many advantages in this process: the digital models are easier and cheaper to modify than their real counterparts. They also can readily be transferred and become available anytime and anywhere by taking advantage of current high-speed telecommunication networks. Owing to these remarkable advantages, reverse engineering is becoming the prevalent technology 
in industrial fields such as computer-aided design and manufacturing (CAD/CAM) (Farin 2002; Varady and Martin 2002) and computer-numerically-controlled (CNC) milling and machining (Patrikalakis and Maekawa 2002) for automotive, aerospace, and ship hull building industries (Pottmann et al. 2005). Another popular field of application is rapid prototyping (the generation of scale models of physical parts from CAD data). A major reason of this popularity is the quick availability of very efficient methods for generating physical prototypes, particularly 3D printing and additive layer manufacturing technology. Due to the fast spread and sharp decline in prices of these technologies, they are a truly emerging field nowadays, particularly regarding the mass customization of personal goods. Surface reconstruction is also very common in biomedical engineering, for the design and manufacturing of prosthesis of different types and customized medical implants. Other relevant examples include computer graphics and animation (e.g., motion capture, inverse kinematics, camera walkthrough rendering, character modeling), entertainment industries, shoe industry (e.g., cutting die making, shoe last design, sole mould design), cultural heritage preservation (Levoy et al. 2000), data processing and scientific visualization (Prasad 2006), virtual and augmented reality (Leu et al. 2005), and medical imaging (computer tomography, magnetic resonance imaging) (Alvino and Yezzi 2004), to mention just a few.

In many cases (particularly, for real-world applications), data points are usually acquired by using 3D laser scanners and other digitizing devices (such as tactile scanners or coordinate measuring machines). The cloud of data points thus obtained is generally affected by measurement noise, irregular sampling, and other artifacts (Barnhill 1992; Patrikalakis and Maekawa 2002; Pottmann et al. 2005). Therefore, the accurate fitting of data requires approximation schemes, in which the fitting surface is not required to pass through all input data points, but just near to them, according to some prescribed distance criteria. In this case, surface reconstruction can be mathematically formulated as an optimization problem. As such, it requires an adequate choice of the fitting functions. A number of choices have been described in the literature (see Sect. 2 for details). Among them, the free-form parametric functions (such as Bézier, B-spline and NURBS) are widely applied in many industrial settings due to their great flexibility and the fact that they can represent smooth shapes with only a few parameters (Barnhill 1992; Jing and Sun 2005; Li et al. 2005; Park 2004; Park and Lee 2007). Although it is possible to obtain good fitting results for a number of shapes, these families of functions are still limited: since they are based on polynomials, they cannot adequately describe some particular shapes, such as the quadrics (see our discussion in Sect. 3.1 for details). As a consequence, there is still a need for more powerful and more general blending functions.

An interesting extension in this regard is given by the rational basis functions, which are mathematically described as the quotient of two polynomials. A remarkable advantage of this rational scheme is that the quadrics and other shapes can be canonically described as rational functions. Unfortunately, this rational approach becomes more difficult than the polynomial one, since new parameters are now introduced into the problem. Consequently, we are confronted with the challenge of obtaining optimal values for many (qualitatively different) parameters, namely, data parameters, poles, and weights. This leads to a very difficult over-determined multivariate nonlinear continuous optimization problem.

\subsection{Aims and structure of the paper}

In a previous paper presented at the conference ICSI 2015, we introduced a method to obtain the rational Bézier curve of a certain degree providing the optimal fit to a cloud of data points (Iglesias and Gálvez 2015). Our scheme was a memetic approach based on the combination of a powerful physics-based algorithm called electromagnetism algorithm and aimed at solving global optimization problems, and a local search procedure. This approach exhibited a very good performance in the previous curve fitting problem, as we could reconstruct difficult shapes such as conics by rational Bézier curves with high accuracy.

The aim of the present paper is to extend our previous method to the (more challenging) case of rational surfaces. This problem is more difficult than it might seem at first sight. The main reasons are:

1. Rational free-form surfaces depend on many different parameters (data parameters, poles, weights, surface degree) that are strongly intertwined each other, leading to a strongly nonlinear continuous optimization problem.

2. It is also a multivariate problem, as it typically involves a large number of unknown variables for a large number of data points, the most common case in real-world applications.

3. The number of parameters of the fitting surface is very critical. On one hand, we expect to obtain an approximating surface with many fewer parameters than the number of data points. This feature is very desirable in order to save computer memory and storage capacity and make the model more manageable. The counterpart is that the problem becomes over-determined. On the other hand, a high number of parameters might lead to over-fitting. As we will shown later on, our method will allows us to determine the best choice of the 
number of parameters by selecting the optimal surface degree (see Sect. 5.3 for details).

4. Finally, the problem is also multimodal, i.e., the leastsquares objective function can exhibit many local optima, meaning that the problem might have several (global and/or local) good solutions.

The structure of this paper is as follows: Sect. 2 summarizes briefly the previous work in the field. Some basic mathematical concepts and definitions and the surface fitting problem with rational Bézier surfaces are described in Sect. 3. The fundamentals and main steps of the memetic electromagnetism algorithm are briefly explained in Sect. 4. Our proposed approach for surface reconstruction with rational Bézier surfaces is described in Sect. 5. To check the performance of our approach, it has been applied to five illustrative examples discussed in Sect. 6. Our experimental results show that the presented method performs very well, being able to replicate the underlying shape of data very accurately. To gain a better insight about our method and further establish its applicability, some additional experiments for comparative work are reported in Sect. 7. The paper closes in Sect. 8 with the main conclusions of this contribution and our plans for future work in the field.

\section{Previous work}

In general, surface reconstruction methods are classified in terms of the available input (2D slices, iso-parametric curves, clouds of points, mixed information, etc.). For instance, authors in Bajaj et al. (2005), Jones and Chen (1994), Meyers et al. (1992) and Park and Kim (1997) address the problem of obtaining a surface model from a set of given cross-sections, a classical problem in medical science, biomedical engineering and CAD/CAM. Other classical input data include iso-parametric curves on the surface (Gordon 1969) and even mixed information, such as scattered points and contours (Fuchs et al. 1977; Maekawa and Ko 2002; Savchenko et al. 1995) or iso-parametric curves and data points (Echevarría et al. 2002; Iglesias et al. 2004; Iglesias and Gálvez 2001).

In most cases, however, the available information about the surface is typically a dense set of (usually unorganized) 3D data points obtained by using some sort of digitizing devices, see e.g. (Gu and Yan 1995; Ma and Kruth 1995). In that case, the reconstructed surface can be described using three different representations providing different levels of accuracy. The simplest one is given by the polygonal meshes, where the data points are used as vertices connected by lines (edges) that work together to create a 3D model, comprised of vertices, edges and faces.
Although it is the coarsest representation, it is also the most popular one because of its simplicity, flexibility and excellent performance with current graphical cards. Surface reconstruction methods with polygonal meshes can be found, for instance, in Levoy et al. (2000) and Prasad (2006) and references therein. The next level is given by the CSG (Constructive Solid Geometry) models, where elementary geometries (such as spheres, boxes, cylinders, or cones) are combined in order to produce more elaborated shapes by applying some simple (Boolean) operators: union, intersection, difference. This methodology works well but presents a low level of flexibility, being severely limited to very simple shapes. The most sophisticated and most accurate level consists of obtaining the real mathematical surface fitting the data points. This issue has been analyzed from several points of view, such as parametric methods (Bolle and Vemuri 1991), subdivision surfaces (Schmitt et al. 1986), function reconstruction (Foley 1990; Sclaroff and Pentland 1991), implicit surfaces (Lim et al. 1995), algebraic surfaces (Pratt 1987), etc.

Artificial neural networks have also been applied to this problem (Gu and Yan 1995; Hoffmann 2005), mostly for arranging the input data in case of unorganized points. After this pre-processing step, any other classical surface reconstruction method operating on organized points is subsequently applied. A work using a combination of neural networks and Partial Differential Equation (PDE) techniques for the parameterization and reconstruction of surfaces from 3D scattered points can be found in Barhak and Fischer (2001). Two previous papers by the authors have also addressed this problem by using functional networks (Gálvez et al. 2007; Iglesias et al. 2004), a powerful generalization of neural networks based on functional equations (Castillo and Iglesias 1997; Castillo et al. 2005). Both works show, however, that the single application of functional networks is still unable to solve the general case. The work in Iglesias et al. (2004) addresses the particular case of B-spline surface reconstruction when some additional information (iso-parametric curves) is available in addition to the data points. The paper in Iglesias and Gálvez (2014) describes the application of a hybrid neuralfunctional network to NURBS surface reconstruction.

Other approaches are based on the application of natureinspired metaheuristic techniques, which have been intensively applied to solve difficult optimization problems that cannot be tackled through traditional optimization algorithms. Genetic algorithms have been applied to this problem in both the discrete version (Sarfraz and Raza 2001) and the continuous version (Gálvez et al. 2012; Yoshimoto et al. 2003). Other metaheuristic approaches applied to this problem include the use of the popular particle swarm optimization technique (Gálvez et al. 2008; Gálvez and Iglesias 2011, 2012), artificial immune systems 
(Gálvez et al. 2013, 2015; Gálvez and Iglesias 2016), firefly algorithm (Gálvez and Iglesias 2013b, c), estimation of distribution algorithms (Zhao et al. 2011), memetic algorithms (Gálvez 2014), and hybrid techniques (Gálvez and Iglesias 2013a), (Sarfraz and Raza 2001).

\section{Description of the problem}

\subsection{Basic concepts and definitions}

A three-dimensional surface is a geometric shape that depends on two independent variables. Mathematically, it can be represented in three different ways: explicit, implicit, and parametric. In explicit form, the surface is given by the expression $z=f(x, y)$, where $x$ and $y$ are the independent variables and $z \in \mathbb{R}$ is a dependent variable associated with the pair $(x, y)$ through $f$. Intuitively, $z$ can be interpreted as the "height" of the surface at the point $(x, y)$, so the explicit representation is often understood as a height map. The explicit representation is arguably the simplest one, but it is also severely limited: it can only associate one value $z$ to each pair $(x, y)$, thus precluding a proper description of closed surfaces and many other useful shapes.

A powerful extension is given by the implicit representation, where the surface is represented by the set of zeros of a function of three variables, as $F(x, y, z)=0$. The implicit representation is more general than the explicit one, since any explicit surface $z=f(x, y)$ can be converted into the implicit form (simply take $F(x, y, z)=z-f(x, y)=0$ ), while the opposite is not true. For instance, surfaces with multiple sheets or closed surfaces such as the sphere can be represented with a single implicit equation but not with an explicit one. In addition, they can represent well any algebraic shape such as the quadrics. Unfortunately, implicit surfaces are very difficult to draw and manipulate, requiring sophisticated and time-consuming methods to handle them.

The most common surface representation in real-world applications is given by the parametric representation. A three-dimensional parametric surface is defined as a mapping $S: D \subset \mathbb{R}^{2} \longrightarrow \mathbb{R}^{3}$, so that any pair $(s, t) \in D$ is transformed into a three-dimensional vector $\mathbf{S}(s, t)=$ $(x(s, t), y(s, t), z(s, t))$. The set $D$ is called the domain of the surface, and $s$ and $t$ are called the surface parameters. If functions $x(s, t), y(s, t)$, and $z(s, t)$ are polynomials in $s$ and $t, S$ is called a polynomial parametric surface. The degree of $S$ in variable $s$ (resp. $t$ ) is the highest degree of polynomials $x(s, t), y(s, t)$, and $z(s, t)$ in $s$ (resp. $t)$.

In this context, a free-form polynomial Bézier surface $\boldsymbol{\Phi}(\tau, \zeta)$ of degree $(\eta, \sigma)$ in $\mathbb{R}^{d}$ is given by:

$$
\boldsymbol{\Phi}(\tau, \zeta)=\sum_{i=0}^{\eta} \sum_{j=0}^{\sigma} \boldsymbol{\Lambda}_{i, j} \phi_{i}^{\eta}(\tau) \phi_{j}^{\sigma}(\zeta)
$$

where $\left\{\boldsymbol{\Lambda}_{i, j}\right\}_{i=0, \ldots, \eta ; j=0, \ldots, \sigma}$ are vector coefficients called the poles, and the functions $\phi_{k}^{\rho}(v)$ are the Bernstein polynomials of index $k$ and degree $\rho$, given by:

$\phi_{k}^{\rho}(v)=\left(\begin{array}{l}\rho \\ k\end{array}\right) v^{k}(1-v)^{\rho-k}$

with $\left(\begin{array}{l}\rho \\ k\end{array}\right)=\frac{\rho !}{k !(\rho-k) !}$ for $k=0, \ldots, \rho$ and where $v$ is the curve parameter, defined on the unit interval $[0,1]$. By convention, $0 !=1$. Note that in this paper vectors are denoted in bold.

The polynomial representation in Eqs. (1)-(2) is not powerful enough to represent a variety of shapes, particularly the quadratic surfaces or quadrics, such as cones, cylinders, ellipsoids, paraboloids, hyperboloids, spheres, and spheroids, which are very important in many different fields. One way to overcome this limitation is to use homogeneous coordinates (see Farin 2002; Piegl and Tiller 1997 for details). The basic idea is to consider the projection of the standard polynomial Bézier surface in $\mathbb{R}^{d+1}$, with new poles $\Lambda_{i, j}^{h}$. The resulting surface in $\mathbb{R}^{d}$ is called a rational Bézier surface. Mathematically, this surface can be described as a quotient of two bivariate polynomial functions. In particular, a free-form rational Bézier surface $\boldsymbol{\Psi}(\tau, \zeta)$ of degree $(\eta, \sigma)$ in $\mathbb{R}^{d}$ is given by:

$\boldsymbol{\Psi}(\tau, \zeta)=\frac{\sum_{i=0}^{\eta} \sum_{j=0}^{\sigma} \omega_{i, j} \boldsymbol{\Lambda}_{i, j} \phi_{i}^{\eta}(\tau) \phi_{j}^{\sigma}(\zeta)}{\sum_{i=0}^{\eta} \sum_{j=0}^{\sigma} \omega_{i, j} \phi_{i}^{\eta}(\tau) \phi_{j}^{\sigma}(\zeta)}$

where $\omega_{j}$ are their scalar weights associated with the poles $\boldsymbol{\Lambda}_{i, j}$. Considering the rational bivariate Bernstein basis functions:

$$
\begin{aligned}
\boldsymbol{\Omega}_{k, l}^{\eta, \sigma}(\tau, \zeta)= & \frac{\omega_{k, l} \phi_{k}^{\eta}(\tau) \phi_{l}^{\sigma}(\zeta)}{\sum_{i=0}^{\eta} \sum_{j=0}^{\sigma} \omega_{i, j} \phi_{i}^{\eta}(\tau) \phi_{j}^{\sigma}(\zeta)} \\
& (k=0, \ldots, \eta ; l=0, \ldots, \sigma)
\end{aligned}
$$

expression (3) can be rewritten as:

$$
\boldsymbol{\Psi}(\tau, \zeta)=\sum_{k=0}^{\eta} \sum_{l=0}^{\sigma} \boldsymbol{\Lambda}_{k, l} \boldsymbol{\Omega}_{k, l}^{\eta, \sigma}(\tau, \zeta) .
$$

Note that weights $w_{i, j}$ are the last coordinates of the homogeneous poles $\boldsymbol{\Lambda}_{k, l}^{h}$. This new set of parameters provides us with additional degrees of freedom for better shape approximation. They also increase the model 
complexity, however, as we introduce a new set of parameters that have to be computed as well.

\subsection{The surface fitting problem}

Let now $\left\{\boldsymbol{\Delta}_{k}\right\}_{k=1, \ldots, \kappa}$ be a set of data points in $\mathbb{R}^{d}$. The problem consists of obtaining the rational Bézier surface, $\boldsymbol{\Psi}(\tau, \zeta)$, of a certain degree $(\eta, \sigma)$ providing the best leastsquares fitting of the data points. This leads to a minimization problem of the least-squares error functional $\mathbf{r}$, related to the weighted sum of squares of the residuals:

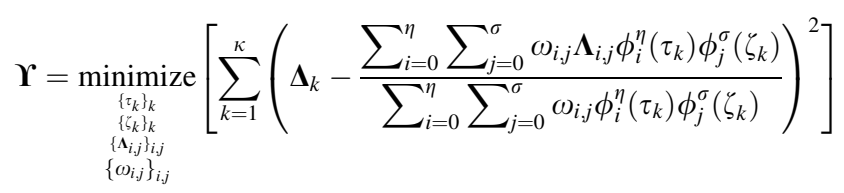

Note that solving this problem (6) requires to compute all parameters (i.e. poles $\boldsymbol{\Lambda}_{i, j}$, weights $\omega_{i, j}$, and parameters $\tau_{k}$ and $\zeta_{k}$ associated with data points $\boldsymbol{\Delta}_{k}$ (for $k=1, \ldots, \kappa, i=0$, $\ldots, \eta, j=0, \ldots, \sigma)$ ) of the approximating surface. It is obvious that, since each blending function in (2) and (4) is nonlinear in $\tau$ and $\zeta$, the system (6) becomes highly non-linear. It is also a continuous problem, since all parameters are real-valued. In other words, we have to deal with a highly nonlinear multivariate continuous optimization problem. The problem is also multimodal, since there can be several optima of the target function. Unfortunately, classical optimization techniques cannot solve this problem in all its generality. Clearly, more powerful strategies are needed. This paper aims at overcoming this limitation by applying the memetic approach described in next section.

\section{Our memetic approach}

During the last two decades, there has been an increasing interest upon the application of soft computing approaches (particularly, metaheuristic techniques) to solve hard optimization problems. Among them, the memetic algorithmsbased on a metaheuristic strategy for global optimization coupled with a local search procedure-have shown a great potential for solving difficult nonlinear optimization problems. Inspired by this idea, in a previous paper we considered a memetic approach combining the electromagnetism algorithm and a local search method to solve the curve fitting problem with rational Bézier curves Iglesias and Gálvez (2015). Our good results in that work encouraged us to extend this method to the case of rational Bézier surfaces, a problem by far more complex than the case for curves. Before describing why this problem is more complex and how we solve it, we describe our memetic approach. It will be subsequently modified to adapt it to our current problem with surfaces, as discussed in Sect. 5.

\subsection{The electromagnetism algorithm}

The electromagnetism algorithm (EMA) is a metaheuristic introduced by Birbil and Fang (2003) for optimization problems. This method utilizes an attraction-repulsion mechanism to move sample points towards optimality. Each point (called particle) is treated as a potential solution and an electric charge is assigned to each particle. Better solutions have stronger charges and each particle has an impact on others through charge. The exact value of the impact is given by a modification of original Coulomb's Law. In EMA, the power of the connection between two particles is proportional to the product of their charges and reciprocal to the distance between them. In other words, the particles with a higher charge will force the movement of other particles in their direction more strongly. Beside that, the best particle in this electromagnetic mechanism will stay unchanged. The charge of each particle relates to the objective function value, which is the subject of optimization. The reader is also referred to Birbil et al. (2004) for a comprehensive study about the convergence of the EMA approach.

The electromagnetism algorithm was originally proposed to study a special class of optimization problems with bounded variables in the form:

$\min \varphi(\boldsymbol{\Theta}) \quad$ such that $\boldsymbol{\Theta} \in[\mathbf{L}, \mathbf{U}]$

where $[\mathbf{L}, \mathbf{U}]:=\left\{\boldsymbol{\Theta} \in \mathbb{R}^{v} / l_{k} \leq \boldsymbol{\Theta}_{k} \leq u_{k}, k=1, \ldots, v\right\}, v$ is the dimension of the problem, $\mathbf{L}=\left\{l_{k}\right\}_{k}$ and $\mathbf{U}=\left\{u_{k}\right\}_{k}$ represent respectively the lower bound and upper bound in $\mathbb{R}^{v}$, and $\varphi(\boldsymbol{\Theta})$ is the function to be optimized. The algorithm consists of four main steps, which are summarized in next paragraphs. The corresponding pseudocode is depicted in Table 1. Note that in this paper vectors are denoted in bold.

\subsubsection{Step 1: Initialization}

In this step, $\mu$ sample points are selected at random from the feasible region, which is an $v$-dimensional hypercube. To this purpose, each coordinate of the sampled point is assumed to be uniformly distributed between the corresponding lower and upper bound. Then, the objective function value of each sampled point is computed, and the point that has the best global value is stored in $\boldsymbol{\Theta}^{\text {best }}$.

\subsubsection{Step 2: Local search}

In this step, a local search is carried out to gather the local information for each point $\Theta^{i}$ and exploit the local minima. 
Table 1 General pseudocode of the electromagnetism algorithm

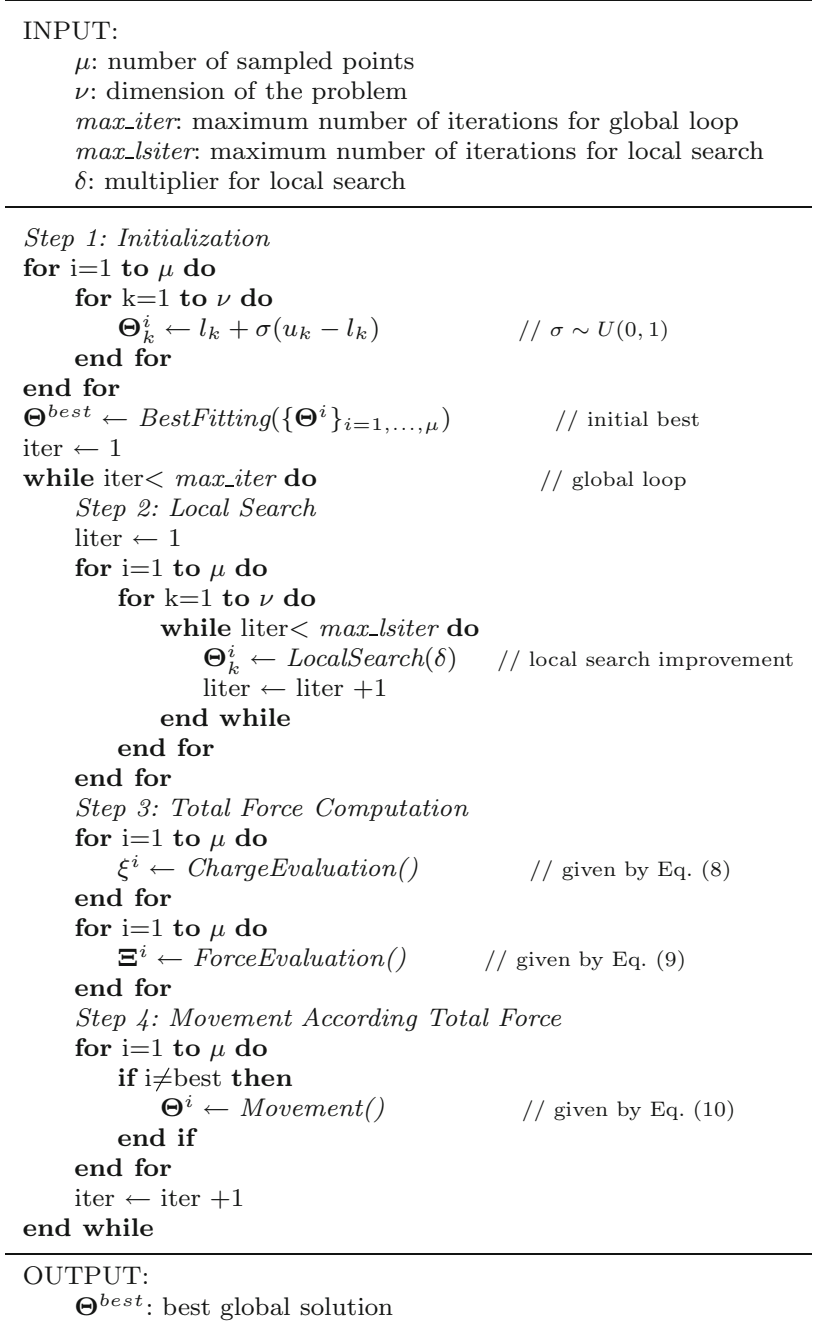

To this aim, a LocalSearch procedure similar to that in Birbil and Fang (2003) is applied. The procedure depends on a multiplier $\delta$ which is used to compute the maximum feasible step length for the local search. The search is performed for each coordinate and for a given number of iterations. In case a better point is obtained (according to the fitness function), the current point is replaced by this new (better) alternative. Note that this procedure does not require any gradient information. Note also that any other local search procedure might be alternatively used, opening the door for other hybridized schemes.

\subsubsection{Step 3: Calculation of total force}

In this step, the vector of the total force exerted on each particle from all other particles is computed. Firstly, a charged-like value $\xi^{i}$ is assigned to each particle. The charge of a particle $i$ determines its power of attraction or repulsion, and is evaluated as:

$\xi^{i}=\exp \left(-v \frac{\varphi\left(\boldsymbol{\Theta}^{i}\right)-\varphi\left(\boldsymbol{\Theta}^{\text {best }}\right)}{\sum_{k=1}^{\mu}\left[\varphi\left(\mathbf{\Theta}^{k}\right)-\varphi\left(\mathbf{\Theta}^{\text {best }}\right)\right]}\right)$

Then, the attraction/repulsion force between two particles is computed using a mechanism inspired in the electromagnetism theory for the charged particles. According to Birbil and Fang (2003), the computation of this force is given by:

$$
\boldsymbol{\Xi}^{i}=\sum_{j=1, j \neq i}^{\mu} \frac{\xi^{i} \xi^{j}}{\left\|\Theta^{j}-\boldsymbol{\Theta}^{i}\right\|^{2}} \begin{cases}\left(\boldsymbol{\Theta}^{j}-\boldsymbol{\Theta}^{i}\right) & \text { if } \varphi\left(\boldsymbol{\Theta}^{j}\right)<\varphi\left(\boldsymbol{\Theta}^{i}\right) \\ \left(\boldsymbol{\Theta}^{i}-\boldsymbol{\Theta}^{j}\right) & \text { if } \varphi\left(\boldsymbol{\Theta}^{j}\right) \geq \varphi\left(\boldsymbol{\Theta}^{i}\right)\end{cases}
$$

Note, however, that the force computed in this way does not follow exactly Coulomb's law, where the force is inversely proportional to the square of the distance. Note also that, unlike electrical charges, there is no sign on the charge of individual particles in Eq. (8). Instead, the direction of a particular force between two particles is determined by comparing the objective function values at such particles. Then, the particle with a better fitness value attracts the other one, while the particle with a worse fitness value repels the other, as indicated by Eq. (9).

\subsubsection{Step 4: Movement according to the total force}

The force vector computed in previous step determines the direction of movement for the corresponding particle according to Eq. (10):

$$
\boldsymbol{\Theta}^{i+1}=\boldsymbol{\Theta}^{i}+\lambda \frac{\Xi^{i}}{\left\|\Xi^{i}\right\|} \circ \boldsymbol{\varphi}
$$

where $\varphi$ is the vector of the feasible movement toward the upper/lower bound for the corresponding dimension, $\lambda$ is a random variable following the uniform distribution, and $\circ$ denotes the Hadamard product.

\subsection{Local optimization method}

The EMA is improved by its hybridization with a local search procedure. We apply the Luus-Jaakola local search method, a heuristic for optimization of real-valued functions (Luus and Jaakola 1973). This method starts with an initialization step, where random uniform values are chosen within the search space. Then, a random uniform value in-between boundary values is sampled for each component. This value is added to the current position of the potential solution to generate a new candidate solution, which replaces the current one only if the value of the 
fitness is improved. Otherwise, the sampling space is multiplicatively decreased by a self-adaptive size of a factor whose strength depends on the difference between consecutive parameters, with the effect of speeding up the convergence to the steady state. This process is repeated iteratively. With each iteration, the neighborhood of the point decreases, so the procedure eventually collapses to a point.

\section{The proposed method}

As discussed above, our problem consists of reconstructing the underlying shape of a cloud of data points by using a rational Bézier surface. This requires to solve a nonlinear least-squares minimization problem while simultaneously minimizing the required number of free parameters. Solving this problem requires to compute four different sets of unknowns: data parameters, poles, weights, and surface degrees. The proposed method to tackle this issue is a hybrid strategy combining a memetic algorithm comprised of a global-search metaheuristic approach (the electromagnetism algorithm described in Sect. 4.1) with a local search method (described in Sect. 4.2), classical methods (least-squares minimization), and information science metrics (Akaike Information Criterion, AIC). It consists of three major steps: data parametrization and weight computation, data fitting, and degree determination. The method can be summarized as follows: we initially set a reasonable range for the surface degrees $(\eta, \sigma)$ (this choice is not critical at all, since their best value will be computed afterwards); then, for each value of this parameter within that range, we apply the memetic approach to perform data parameterization and weight computation in Sect. 5.1. Then, data fitting is performed via least-squares to compute the poles of the surface in Sect. 5.2. Finally, AIC is applied to obtain the best values for $(\eta, \sigma)$ in Sect. 5.3. All these steps are explained in detail in next sections.

\subsection{Data parameterization and weight computation}

The goal of this step is to obtain an association between the set of parameters $\left\{\tau_{k}, \zeta_{k}\right\}_{k=1}^{\kappa}$ and the data points $\left\{\boldsymbol{\Delta}_{k}\right\}$ as well as to compute the best values for the weights. It is performed by using the memetic approach described in Sect. 4. In our problem, the parameter vectors $\left\{\tau_{k}\right\}$ and $\left\{\zeta_{k}\right\}$ and weights $\omega_{i, j}$ are considered particles, and the fitness function is given by Eq. (6). All data parameters are initialized with random numbers within the hypercube $[0,1]^{\kappa} \subset \mathbb{R}^{\kappa}$. The weights are randomly initialized within the search domain $(0,100]^{(\eta+1) \times(\sigma+1)}$. For computational efficiency, we store the particles as the super-vector $\mathcal{V}=\{\mathcal{T}, \mathcal{Z}, \mathcal{W}\}$, where $\mathcal{T}=$ $\left\{\tau_{k}\right\}, \mathcal{Z}=\left\{\zeta_{k}\right\}, \mathcal{W}=\operatorname{vec}\left(\left(\left\{\omega_{i, j}\right\}\right)^{T}\right), \operatorname{vec}($.$) denotes the$ vectorization of a matrix (the linear transformation which converts the matrix into a column vector by stacking its columns on top of one another) and $(.)^{T}$ denotes the transpose of a vector or matrix. We also set the number of iterations for the global loop and the local search. Regarding our stopping criterion, we run our method until there is no improvement after 30 consecutive iterations.

\subsection{Data fitting}

Using the parameterization and weights calculated in previous step, the surface poles $\left\{\mathbf{b}_{j}\right\}_{j=0}^{n}$ are now computed. Using Eqs. (3), (6) can be rewritten as:

$\mathbf{D}=\mathbf{R} \cdot \boldsymbol{\Lambda}$

where $\mathbf{D}=\operatorname{vec}\left(\left(\left\{\boldsymbol{\Delta}_{k}\right\}\right)^{T}\right), \boldsymbol{\Lambda}=\operatorname{vec}\left(\left(\left\{\boldsymbol{\Lambda}_{i, j}\right\}\right)^{T}\right)$, and where the matrix $\mathbf{R}$ represents the vector of all rational basis functions given by Eq. (4) at the best parameter values, given by:

$$
\mathbf{R}=\operatorname{vec}\left[\left(\left\{\operatorname{vec}\left(\left(\left\{\mathbf{\Omega}_{k, l}^{\eta, \sigma}\left(\tau_{k}, \zeta_{k}\right)\right\}_{k}\right)^{T}\right)\right\}_{i, j}\right)^{T}\right]
$$

Note that vector $\mathbf{D}$ is of length $\kappa$ while vector $\boldsymbol{\Lambda}$ is of length $(\eta+1) \times(\sigma+1)$, so the system (11) is over-determined, meaning that no analytical solution can be obtained. Premultiplication of both sides of (11) by $\mathbf{R}^{T}$ gives:

$\mathbf{R}^{T} . \mathbf{D}=\mathbf{R}^{T} . \mathbf{R} \cdot \boldsymbol{\Lambda}$

which can now be solved numerically by a classical linear least-squares minimization. From a computational point of view, it can be obtained by either LU decomposition or singular value decomposition (SVD). In this work, we choose SVD because it returns the best answer of this leastsquares problem. To this purpose, SVD computes the generalized inverse (also known as Moore-Penrose pseudo-inverse) of $\mathbf{R}$, denoted by $\mathbf{R}^{+}$. Then, $\boldsymbol{\Lambda}=\mathbf{R}^{+}$.D is the least-squares solution of this data fitting problem.

\subsection{Degree determination}

Previous steps assumed a given degree $(\eta, \sigma)$ for the rational Bézier fitting surface. However, the optimal degree is a problem-dependent issue, so we need a method to compute it. This is not an easy task, since it requires to get an adequate trade-off between two competing factors: the accuracy of the fitting and the complexity of the model. Essentially, increasing the number of poles increases the accuracy of the model, because we have more degrees of freedom (i.e., additional poles and weights) to adjust the data. However, this process also increases the complexity 
of the model and can eventually lead to over-fitting. In order to prevent these undesirable effects, in this paper we use the Akaike Information Criterion (AIC) for the resulting model. The AIC is an information criterion providing a good compromise between data-fidelity and model complexity Akaike (1973), Akaike (1974). Such a trade-off is attained by introducing an additional term into the target function to penalize more complex models. As a result, the resulting fitness function becomes:

$A I C=\kappa \log (\Upsilon)+2 \xi$

where $\xi$ accounts for the number of free parameters of the model. Note that for fixed values of $\kappa$ and $\xi$, the AIC behaves like a classical error function. If we fix a value of $\kappa$ and $\Upsilon$, the criterion penalizes those models with a higher number of parameters. Therefore, AIC constitutes a powerful procedure to compute the optimal value for the degree of the fitting surface while keeping the model as simple as possible. The best choice always corresponds to the parameter values $(\eta, \sigma)$ providing the smallest value for the AIC.

\section{Experimental results}

The method described in previous section has been applied to several examples. In this section we describe only five of them to keep the paper in manageable size. We think, however, that the examples reported here will be useful to readers to determine the good applicability of our method to this problem. The examples correspond to three realworld free-form shapes and two academic examples with several changes of curvature. In all cases, data points are affected by noise and irregular sampling, so they actually replicate the usual conditions of real-world applications at full extent.

First example corresponds to the upper surface of a mobile phone model commercially available years ago. From it, a set of 2347 data points have been extracted by manual operation with a domestic low-price (but highly noisy) scanner device. This cloud of points is shown in Fig. 1 (top). We applied our method to this example. The best fitting surface we obtained is displayed in Fig. 1 (middle). It corresponds to a rational Bézier surface of degree $(19,17)$. We also combine both pictures in Fig. 1 (bottom) for the sake of comparison. As the reader can see, the surface fits the cloud of data points very well, even although the cloud is affected by measurement noise. In this case, the signal-to-noise $(S N R)$ ratio is $S N R=25.5$, corresponding to a low-intensity noise for our problem. This example is more complex than it appears at first sight because the range for the vertical component is very small in comparison with the horizontal axis. Still, the surface
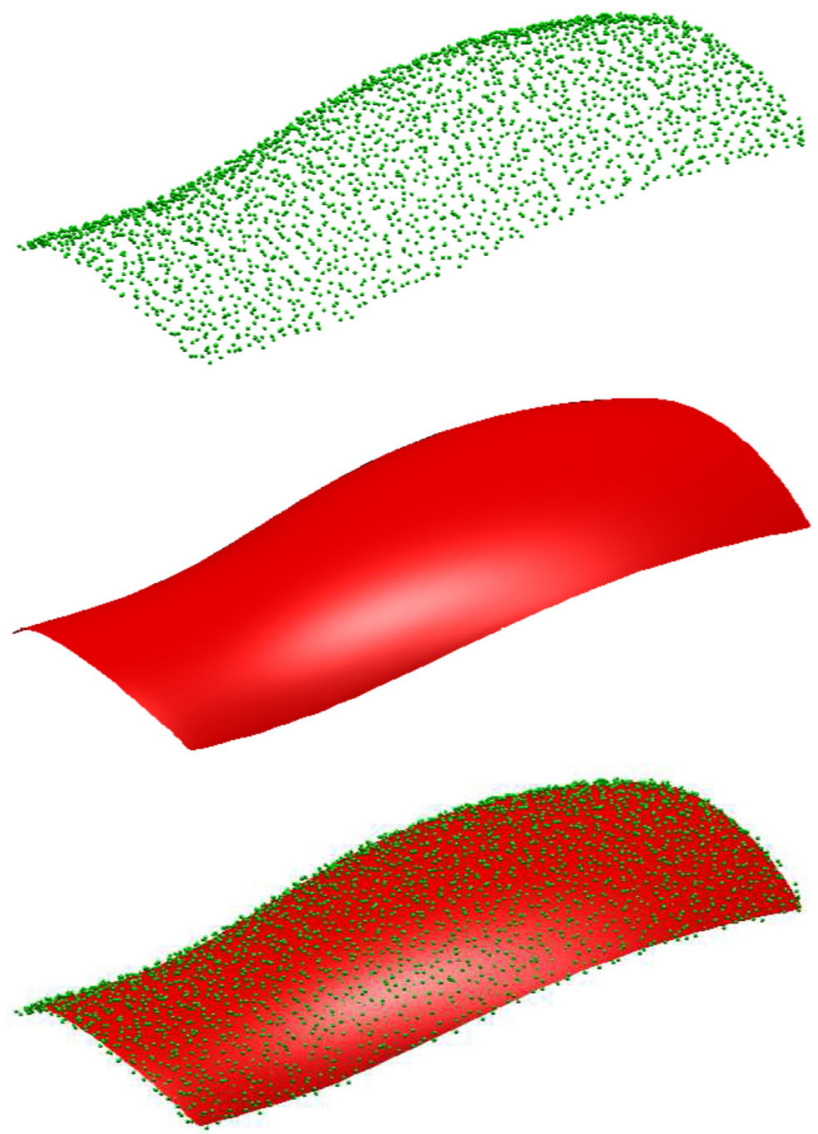

Fig. 1 Application of our memetic electromagnetism algorithm to the mobile phone surface example: top cloud of data points; middle best fitting rational Bézier surface; bottom combination of the cloud of data points and their best fitting surface for better visualization

has a complicated shape requiring a high degree for optimal fitting.

Second example corresponds to the half part of a teapot spout, represented in Fig. 2 (the description of the pictures in this figure is similar to the previous example-but placed from left to right - and will be omitted here to avoid redundant material). In this example, we consider a set of 2074 data points, which are affected by a noise of signalto-ratio $S N R=13.2$, corresponding to noise of medium intensity for our problem. Best fitting surface, displayed in Fig. 2(middle) corresponds to the case of degree $(3,3)$.

Third example corresponds to the closed shape of an apple, represented in Fig. 3. In this example, we consider a set of 2918 data points affected by a noise of signal-to-ratio $S N R=15$, corresponding to noise of relatively medium intensity for our problem. Best fitting surface corresponds to the case of degree $(8,12)$. This example is particularly challenging because it contains a number of very difficult features. On one hand, it is a closed surface in vertical and horizontal directions. In addition, it contains some turning 


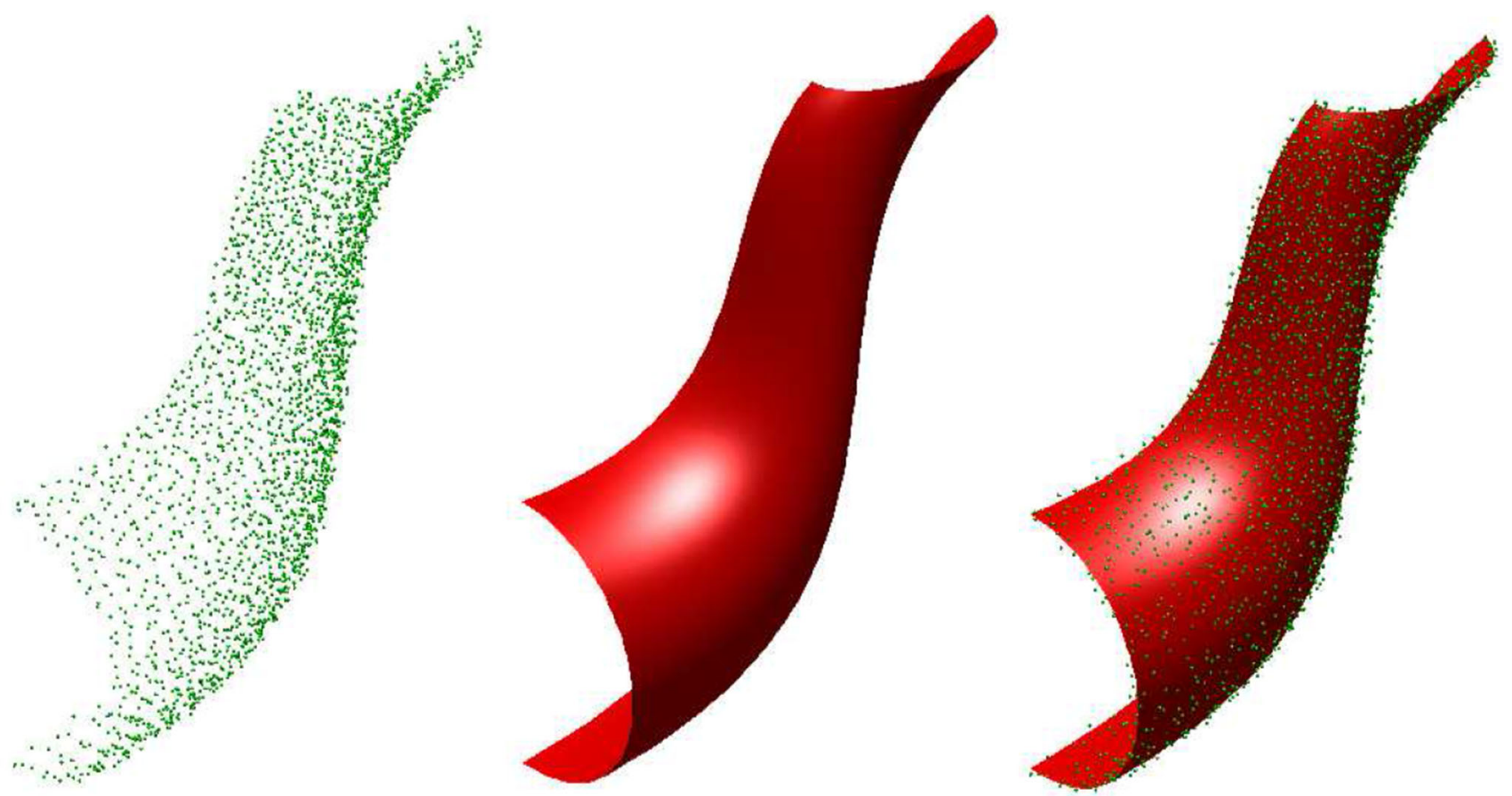

Fig. 2 Application of our memetic electromagnetism algorithm to the teapot spout surface example: left cloud of data points; middle best fitting rational Bézier surface; right combination of the cloud of data points and their best fitting surface for better visualization
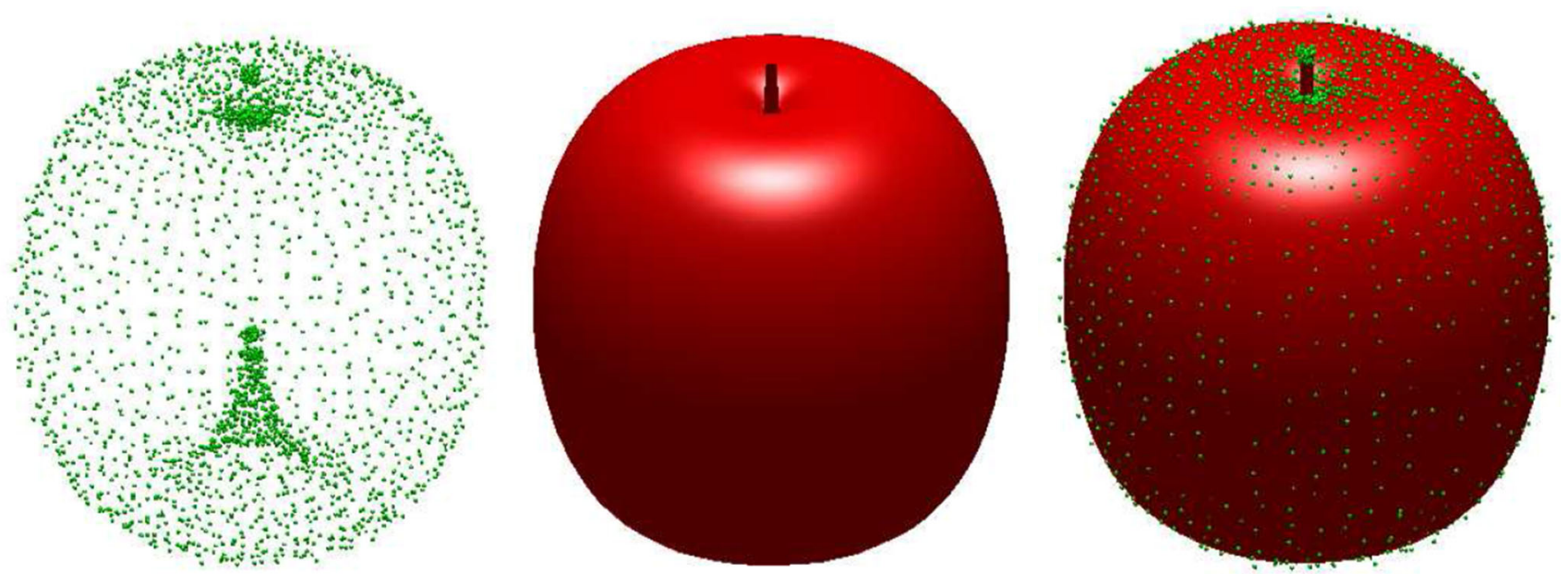

Fig. 3 Application of our memetic electromagnetism algorithm to the apple surface example: left cloud of data points; middle best fitting rational Bézier surface; right combination of the cloud of data points and their best fitting surface for better visualization

points, where the surface is no longer differentiable. It is also greatly affected by irregular sampling. As the reader can see in Fig. 3(top), there is a significant accumulation of data points in the north and south poles of the shape. Finally, we also include the apple tail in our shape. This differs from the classical approaches in computer graphics where the apple and its tail are considered different objects and hence, represented with different surfaces. Instead, in our model we are able to describe the whole apple with just a single rational surface. In our opinion, this is a very remarkable feature with potential applications in several fields. In spite of all these challenging features, the method performs very well, being able to replicate the original shape with high accuracy. Note, for instance, the good visual matching between the original data points and the approximating surface.

The fourth and fifth examples (labelled as Surface IV and Surface $V$ ) are represented in left and right columns of Fig. 4, respectively. Instead of real-world shapes, they are academic examples included here to analyze the 
performance of our approach against several changes of curvature. To this purpose, both surfaces have been carefully chosen so that they exhibit several peaks and valleys and, hence, several changes of curvature in different directions. The fourth example consists of a set of 2601 data points affected by noise of $S N R=22$. Best fitting surface, displayed in Fig. 4(left-middle), corresponds to the case of degree $(8,9)$. The fifth example is given by a set of
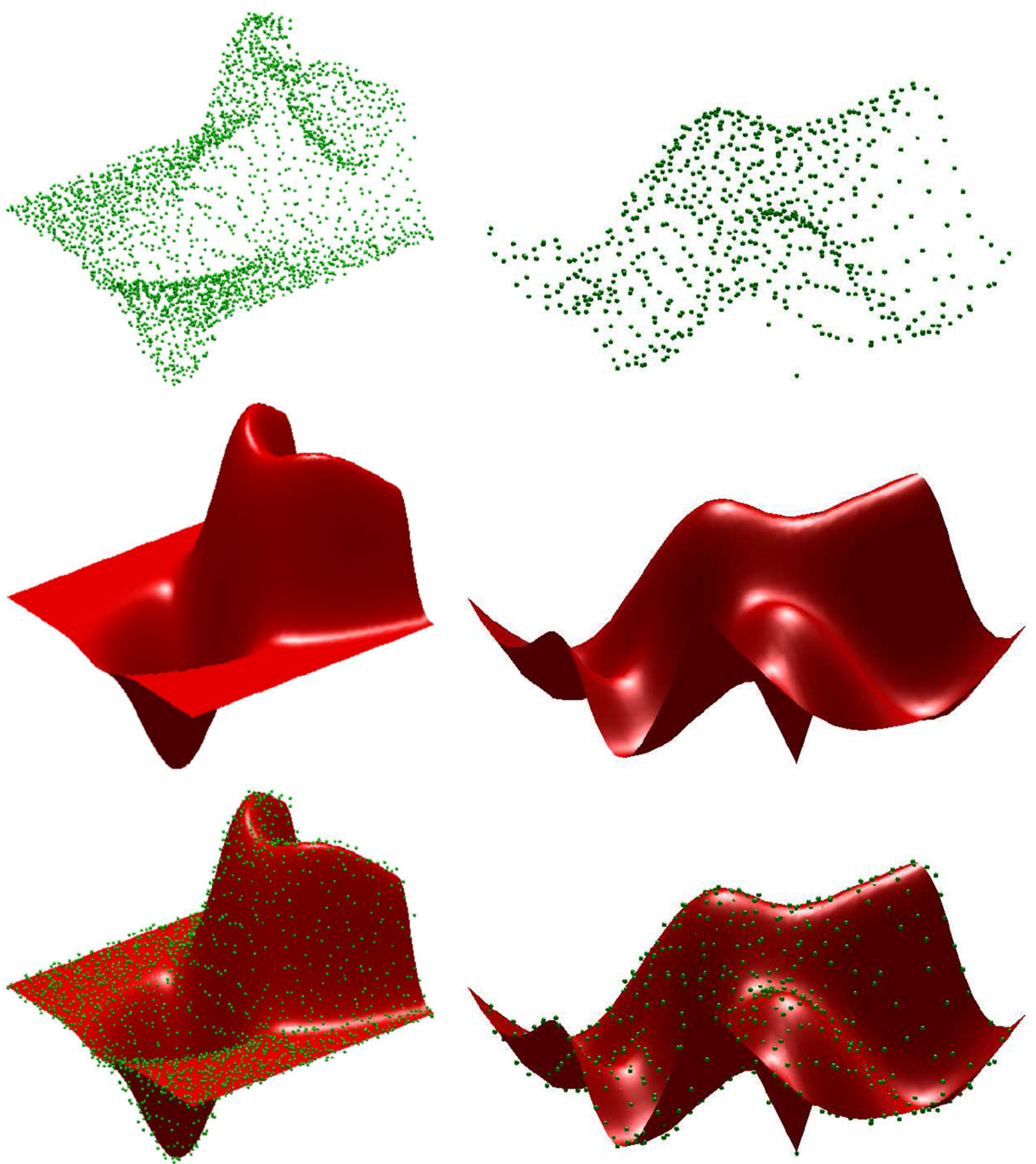

Fig. 4 Application of our memetic electromagnetism algorithm to two academic examples (labelled as Surface $I V$ and Surface $V$ and displayed in left and right columns, respectively): top cloud of data points; middle best fitting rational Bézier surface; bottom combination of the cloud of data points and their best fitting surface for better visualization 
634 noisy data points with $S N R=28$. In this case, the best fitting surface has degree $(7,6)$ [see Fig. 4(right-middle)]. As the reader can see, both surfaces replicate the underlying shape of the data points with very good accuracy even in presence of noise. This fact is particularly noticeable in last row of Fig. 4, where the best fitting surface and the cloud of data points are combined into a single picture for both cases. These examples show that our method performs vey well even in cases of shapes exhibiting several peaks and valleys leading to several changes of curvature.

This good visual behavior in all cases is confirmed by our numerical results, shown in Table 2. The different examples of the benchmark in this paper are arranged in rows. For each example, the table reports the following items (in columns): number of data points, degrees of freedom of the corresponding optimization problem, degree of the best fitting surface according to the $A I C$ criterion, and mean and best value of the error functional $\Upsilon$ given by Eq. (6). Since this functional does not take into account the number of data points, we also compute the RMSE (root-mean squared error), given by:

$$
R M S E=\sqrt{\frac{\Upsilon}{\kappa}}
$$

The mean and best RMSE for our examples is reported in the last two columns of Table 2. All results in the table have been obtained from 50 independent executions to avoid spurious results derived from the stochasticity of the process.

A simple observation of our results in Table 2 shows the good performance of the method for all instances in our benchmark. Mean values for $\Upsilon$ are of order $10^{-2}$ while the best fitting surfaces are of order $10^{-2}$ to $10^{-3}$. Similarly, the mean and best RMSE are of order $10^{-3}$ in all cases. We remark that these good results are obtained for challenging shapes (particularly, the third one is extremely difficult) and under very unfavorable conditions, such as noisy data and irregular sampling. These two adverse features put a strong limitation in the fitting quality, but at the same time, are very common in real-world situations. This means that our method can be directly applied to real-world problems without the need of further pre/post-processing.

Regarding the implementation issues, all computations in this paper have been performed on a $2.6 \mathrm{GHz}$. Intel Core i7 processor with $8 \mathrm{~GB}$. of RAM. The source code has been implemented by the authors in the native programming language of the popular scientific program Matlab, version 2013b.

\section{Comparative work}

We have carried out some additional experiments for comparative purposes to gain a better insight about our method and further establish its applicability. Unfortunately, no other method has been described in the literature so far to address the issue of surface reconstruction with rational Bézier surfaces, a clear indication of the originality of our approach. In our comparison we consider four variations of our memetic approach, depending on whether or not the two most relevant features of our method are considered:

1. computation of weights, leading to either a polynomial scheme (no weights allowed) or a rational scheme, and

2. hybridization with a local search method.

As a result, four different schemes are obtained:

I. Polynomial scheme without local search;

II. Polynomial scheme with local search;

III . Rational scheme without local search;

IV. Rational scheme with local search.

These schemes have been applied to the five examples in our benchmark by following a similar procedure to that in previous section. The obtained results are reported in Tables 3, 4, 5, 6 and 7, respectively.

Some important conclusions can be drawn from our results in those tables. First of all, the method proposed in this paper (scheme IV) outperforms the other three methods for all instances in our benchmark. This is not a surprising fact, since the rational approach is actually an extension of the polynomial one, based on allowing new
Table 2 Fitting errors for the examples (arranged in rows) used in this paper

\begin{tabular}{llllllll}
\hline Surface & $\kappa$ & DOFs & Degree & $\Upsilon$ (mean) & $\Upsilon$ (best) & $R M S E$ (mean) & $R M S E$ (best) \\
\hline Mobile phone & 2347 & 6022 & $(19,17)$ & $1.5653 \mathrm{e}-2$ & $9.1754 \mathrm{e}-3$ & $2.5825 \mathrm{e}-3$ & $1.9772 \mathrm{e}-3$ \\
Teapot spout & 2074 & 4136 & $(3,3)$ & $5.1923 \mathrm{e}-2$ & $2.6891 \mathrm{e}-2$ & $5.0035 \mathrm{e}-3$ & $3.6008 \mathrm{e}-3$ \\
Apple & 2918 & 6320 & $(8,12)$ & $6.5276 \mathrm{e}-2$ & $4.3365 \mathrm{e}-2$ & $4.7297 \mathrm{e}-3$ & $3.8550 \mathrm{e}-3$ \\
Surface IV & 2601 & 5474 & $(8,9)$ & $4.9375 \mathrm{e}-2$ & $3.4341 \mathrm{e}-2$ & $4.3474 \mathrm{e}-3$ & $3.6331 \mathrm{e}-3$ \\
Surface V & 634 & 1420 & $(7,6)$ & $5.0964 \mathrm{e}-2$ & $3.9832 \mathrm{e}-2$ & $8.9610 \mathrm{e}-3$ & $7.9246 \mathrm{e}-3$ \\
\hline
\end{tabular}

The following items are reported (in columns): number of data points, degrees of freedom, degree of the best fitting surface, mean and best $\Upsilon$ error, and mean and best RMSE from 50 executions 
Table 3 Fitting errors of the four variations of our memetic approach applied to the mobile phone example

\begin{tabular}{llllll}
\hline Method & Degree & $\Upsilon$ (mean) & $\Upsilon$ (best) & $R M S E$ (mean) & RMSE (best) \\
\hline Scheme I & $(26,23)$ & $8.2538 \mathrm{e}-1$ & $5.8357 \mathrm{e}-1$ & $1.8753 \mathrm{e}-2$ & $1.5768 \mathrm{e}-2$ \\
Scheme II & $(24,21)$ & $5.9635 \mathrm{e}-1$ & $4.1636 \mathrm{e}-1$ & $1.5940 \mathrm{e}-2$ & $1.3319 \mathrm{e}-2$ \\
Scheme III & $(19,17)$ & $2.7149 \mathrm{e}-2$ & $1.7301 \mathrm{e}-2$ & $3.4011 \mathrm{e}-3$ & $2.7150 \mathrm{e}-3$ \\
Scheme IV & $(19,17)$ & $1.5653 \mathrm{e}-2$ & $9.1754 \mathrm{e}-3$ & $2.5825 \mathrm{e}-3$ & $1.9772 \mathrm{e}-3$ \\
\hline
\end{tabular}

The following items are reported (in columns): degree of the best fitting surface, mean and best $\Upsilon$ error, and mean and best $R M S E$

\begin{tabular}{llllll}
\hline Method & Degree & $\Upsilon$ (mean) & $\Upsilon$ (best) & $R M S E$ (mean) & $R M S E$ (best) \\
\hline Scheme I & $(3,3)$ & $6.1198 \mathrm{e}-2$ & $3.7854 \mathrm{e}-2$ & $5.4320 \mathrm{e}-3$ & $4.2722 \mathrm{e}-3$ \\
Scheme II & $(3,3)$ & $6.0726 \mathrm{e}-2$ & $3.6443 \mathrm{e}-2$ & $5.4110 \mathrm{e}-3$ & $4.1918 \mathrm{e}-3$ \\
Scheme III & $(3,3)$ & $5.4327 \mathrm{e}-2$ & $2.7310 \mathrm{e}-2$ & $5.1180 \mathrm{e}-3$ & $3.6287 \mathrm{e}-3$ \\
Scheme IV & $(3,3)$ & $5.1923 \mathrm{e}-2$ & $2.6891 \mathrm{e}-2$ & $5.0035 \mathrm{e}-3$ & $3.6008 \mathrm{e}-3$ \\
\hline
\end{tabular}

The following items are reported (in columns): degree of the best fitting surface, mean and best $\Upsilon$ error, and mean and best $R M S E$

\begin{tabular}{llllll}
\hline Method & Degree & $\Upsilon$ (mean) & $\Upsilon$ (best) & $R M S E$ (mean) & $R M S E$ (best) \\
\hline Scheme I & $(26,33)$ & $6.4331 \mathrm{e}-1$ & $6.0102 \mathrm{e}-1$ & $1.4848 \mathrm{e}-2$ & $1.4351 \mathrm{e}-2$ \\
Scheme II & $(24,32)$ & $2.0623 \mathrm{e}-1$ & $1.4716 \mathrm{e}-1$ & $8.4068 \mathrm{e}-3$ & $7.1015 \mathrm{e}-3$ \\
Scheme III & $(8,12)$ & $6.8311 \mathrm{e}-2$ & $4.9102 \mathrm{e}-2$ & $4.8384 \mathrm{e}-3$ & $4.1021 \mathrm{e}-3$ \\
Scheme IV & $(8,12)$ & $6.5276 \mathrm{e}-2$ & $4.3365 \mathrm{e}-2$ & $4.7297 \mathrm{e}-3$ & $3.8550 \mathrm{e}-3$ \\
\hline
\end{tabular}

The following items are reported (in columns): degree of the best fitting surface, mean and best $\Upsilon$ error, and mean and best RMSE
Table 5 Fitting errors of the four variations of our memetic approach applied to the apple example
Table 4 Fitting errors of the four variations of our memetic approach applied to the teapot spout example free variables (the weights) to be used for better fitting. As expected, the addition of these extra degrees of freedom improves the efficiency of the process in all cases. Another interesting conclusion is that the hybridization with a local search also improves the fitting errors, but not dramatically. Clearly, the computation of weights is by far a more important factor than the local search. This becomes evident by the simple observation of the degree of the best fitting surface (second column in Tables 3, 4, 5, 6, 7). In general, even although the fitting errors always improve when local search is included, the best degree does not change with the addition of the local search in the rational case (from Scheme III to IV) or changes slightly in the polynomial case (from Scheme I to II). On the contrary, the computation of weights has a great impact on the quality of fitting (see, for instance, the significant improvement from Scheme I to III, or from Scheme II to IV). We also remark that, even although the fitting errors are still worse, the best fitting errors for the polynomial case have been obtained for higher degrees than for the rational case. This means that any decreasing of fitting errors in the polynomial case is reached at the expense of introducing extra degrees of freedom by increasing the polynomial degree of the fitting surface. This is not true for the rational case, where the weights are the source for extra degrees of freedom instead.

A remarkable observation from our experiments is that although both features (weights and local search) improve the fitting process in all cases, the improvement rate of the fitting errors is still problem-dependent. For instance, it is about one order of magnitude for the first, third, fourth, and fifth examples (Tables 3, 5, 6, 7, respectively), while the improvement is modest for the second example. This means that this example can also be reconstructed with a polynomial scheme without too much loss of quality. In other words, the improvement rate is strongly related to the geometric shape of the underlying surface, a kind of measure of the "rationality" of the shape. The better the improvement rate, the more similar the shape to a rational surface.

To summarize, our memetic approach based on a rational scheme with local search improves all other combinations of polynomial or rational schemes with or without local search for all instances in our benchmark. This fact is not accidental; instead, it applies to any example and can be explained by the superior ability of rational functions to replicate very complicated shapes compared to strictly polynomial functions. 
Table 6 Fitting errors of the four variations of our memetic approach applied to the Surface IV example

\begin{tabular}{llllll}
\hline Method & Degree & $\Upsilon$ (mean) & $\Upsilon$ (best) & $R M S E$ (mean) & $R M S E$ (best) \\
\hline Scheme I & $(11,14)$ & $3.0149 \mathrm{e}-1$ & $2.5447 \mathrm{e}-1$ & $1.0766 \mathrm{e}-2$ & $9.8911 \mathrm{e}-3$ \\
Scheme II & $(11,13)$ & $1.8767 \mathrm{e}-1$ & $1.3228 \mathrm{e}-1$ & $8.4942 \mathrm{e}-3$ & $7.1314 \mathrm{e}-3$ \\
Scheme III & $(9,9)$ & $6.0193 \mathrm{e}-2$ & $5.7148 \mathrm{e}-2$ & $4.8106 \mathrm{e}-3$ & $4.6873 \mathrm{e}-3$ \\
Scheme IV & $(8,9)$ & $4.9375 \mathrm{e}-2$ & $3.4341 \mathrm{e}-2$ & $4.3474 \mathrm{e}-3$ & $3.6331 \mathrm{e}-3$ \\
\hline
\end{tabular}

The following items are reported (in columns): degree of the best fitting surface, mean and best $\Upsilon$ error, and mean and best RMSE

\begin{tabular}{llllll}
\hline Method & Degree & $\Upsilon$ (mean) & $\Upsilon$ (best) & RMSE (mean) & RMSE (best) \\
\hline Scheme I & $(12,11)$ & $4.6118 \mathrm{e}-1$ & $4.3109 \mathrm{e}-1$ & $2.9697 \mathrm{e}-2$ & $2.6075 \mathrm{e}-2$ \\
Scheme II & $(11,11)$ & $4.3285 \mathrm{e}-1$ & $3.9312 \mathrm{e}-1$ & $2.6129 \mathrm{e}-2$ & $2.4901 \mathrm{e}-2$ \\
Scheme III & $(7,6)$ & $5.1783 \mathrm{e}-2$ & $4.0713 \mathrm{e}-2$ & $9.0375 \mathrm{e}-3$ & $8.0134 \mathrm{e}-3$ \\
Scheme IV & $(7,6)$ & $5.0964 \mathrm{e}-2$ & $3.9832 \mathrm{e}-2$ & $8.9610 \mathrm{e}-3$ & $7.9246 \mathrm{e}-3$ \\
\hline
\end{tabular}

The following items are reported (in columns): degree of the best fitting surface, mean and best $\Upsilon$ error, and mean and best $R M S E$
Table 7 Fitting errors of the four variations of our memetic approach applied to the Surface V example

\section{Conclusions and future work}

This paper is an extension of a previous paper published in the conference ICSI 2015, held in Beijing (China) in June 2015 Iglesias and Gálvez (2015). In that paper, we introduced a new memetic electromagnetism method for finite approximation with rational Bézier curves. The method was based on a memetic approach combining a powerful metaheuristic method for global optimization (the electromagnetism algorithm) to obtain a very good approximation of the optimal solution and a local search procedure (the Luus-Jaakola local search method) for further solution refinement.

In this work, a similar approach is applied to solve the problem of surface approximation from noisy data points with rational Bézier surfaces. This problem is by far more complex than the case for curves, not only because the number of free variables is much larger than the case of curves but also because the rational surfaces are not tensorproduct surfaces. This means that it is not possible to compute the surface as a combination of a net of curves in the two parametric directions. Given a set of data points, the method computes all relevant parameters (poles, weights, and data parameters) of the rational Bézier fitting surface as the solution of a difficult over-determined continuous multivariate nonlinear optimization problem. Moreover, we are able to determine the optimal value for the surface degree by using the Akaike information criterion. This allows us to obtain the best fitting surface to the cloud of data points. In addition, in this work we deal with data points subjected to measurement noise and irregular sampling. These features introduce extra difficulties in the problem. The counterpart is that we can replicate the usual conditions of real-world applications, meaning that our results are applicable without further pre/post-processing. Our method has been applied to a benchmark of five illustrative examples (three real-world free-form shapes and two academic examples) exhibiting challenging features such as closed shapes, turning points, and several changes of curvature. Our experimental results show that the method performs very well, and it can recover the underlying shape of surfaces with very good accuracy. Additional experiments reported in this paper show that our proposal based on a rational scheme with local search improves all other combinations of polynomial or rational schemes with or without local search for all instances in our benchmark. This remarkable feature of our approach can be explained by the superior ability of rational functions to replicate very complicated shapes compared to strictly polynomial functions. From this point of view, this paper opens an interesting and promising line of research based on the replacement of the classical polynomial schemes for data fitting by more sophisticated fitting functions.

Future work includes the extension of this method to other families of functions, such as the B-splines. We are also interested to analyze the application of this method to some industrial processes and other interesting real-world problems for which the polynomial approximation is not good enough, thus expanding the potential range of applications by including more difficult shapes.

Acknowledgments This research is kindly supported by the Computer Science National Program of the Spanish Ministry of Economy 
and Competitiveness, Project \#TIN2012-30768, Toho University, and the University of Cantabria. The authors are particularly grateful to the Department of Information Science of Toho University for all the facilities given to carry out this work. We also thank the Editor and the two anonymous reviewers who helped us to improve our paper with several constructive comments and suggestions.

\section{References}

Akaike H (1973) Information theory and an extension of the maximum likelihood principle. In: Petrov BN, Csaki F (eds) Second international symposium on information theory. Akademiai Kiado, Budapest, pp 267-281

Akaike H (1974) A new look at the statistical model identification. IEEE Trans Autom Control 19(6):716-723

Alvino CV, Yezzi AJ (2004) Tomographic reconstruction of piecewise smooth images. In: Proceedings of computer vision and pattern recognition-CVPR'04. IEEE Computer Society Press, Los Alamitos, CA, vol 1, pp 576-581

Bajaj C, Bernardini F, Xu G (1995) Automatic reconstruction of surfaces and scalar fields from 3D scans. In: Proceedings of SIGGRAPH'95, pp 109-118

Barhak J, Fischer A (2001) Parameterization and reconstruction from 3D scattered points based on neural network and PDE techniques. IEEE Trans. Vis Comput Graph 7(1):1-16

Barnhill RE (1992) Geometric Processing for Design and Manufacturing. SIAM, Philadelphia

Birbil SI, Fang SC (2003) An electromagnetism-like mechanism for global optimization. J Global Optim 25:263-282

Birbil SI, Fang SC, Sheu RL (2004) On the convergence of a population-based global optimization algorithm. J Global Optim 30:301-318

Bolle RM, Vemuri BC (1991) On three-dimensional surface reconstruction methods. IEEE Trans Pattern Anal Mach Intell 13(1):1-13

Castillo E, Iglesias A (1997) Some characterizations of families of surfaces using functional equations. ACM Trans Graph 16(3):296-318

Castillo E, Iglesias A, Ruiz-Cobo R (2005) Functional equations in applied sciences. Elsevier Science, Amsterdam

Cox MG (1993) Algorithms for spline curves and surfaces. In: Piegl L (ed) Fundamental developments of computer-aided geometric design. Academic Press, London, San Diego, pp 51-76

Draper NR, Smith H (1998) Applied regression analysis, 3rd edn. Wiley-Interscience, New York

Eck M, Hoppe H (1996) Automatic reconstruction of B-Spline surfaces of arbitrary topological type. In: Proceedings of SIGGRAPH'96, pp 325-334

Echevarría G, Iglesias A, Gálvez A (2002) Extending neural networks for B-spline surface reconstruction. In: Lectures notes in computer science, vol 2330, pp 305-314

Farin G (2002) Curves and surfaces for CAGD, 5th edn. Morgan Kaufmann, San Francisco

Foley TA (1990) Interpolation to scattered data on a spherical domain. In: Mason JC, Cox MG (eds) Algorithms for approximation II. Chapman and Hall, London, New York, pp 303-310

Forsey DR, Bartels RH (1995) Surface fitting with hierarchical splines. ACM Trans Graph 14:134-161

Franke RH, Schumaker LL (1986) A bibliography of multivariate approximation. In: Chui CK, Schumaker LL, Utreras FI (eds) Topics in multivariate approximation. Academic Press, New York

Fuchs H, Kedem ZM, Uselton SP (1977) Optimal surface reconstruction from planar contours. Commun ACM 20(10):693-702
Gálvez A, Iglesias A (2011) Efficient particle swarm optimization approach for data fitting with free knot B-splines. Comput Aided Des 43(12): 1683-1692

Gálvez A, Iglesias A (2012) Particle swarm optimization for nonuniform rational B-spline surface reconstruction from clouds of 3D data points. Inf Sci 192(1):174-192

Gálvez A, Iglesias A (2013a) A new iterative mutually-coupled hybrid GA-PSO approach for curve fitting in manufacturing. Appl Soft Comput 13(3):1491-1504

Gálvez A, Iglesias A (2013b) Firefly algorithm for polynomial Bézier surface parameterization. J Appl Math, Article ID 237984

Gálvez A, Iglesias A (2013c) From nonlinear optimization to convex optimization through firefly algorithm and indirect approach with applications to CAD/CAM. Sci World J, Article ID 283919

Gálvez A, Iglesias A (2014) New memetic self-adaptive firefly algorithm for continuous optimization. Int $\mathrm{J}$ Bio-Inspir Comput (in press)

Gálvez A, Iglesias A (2016) Particle-based meta-model for continuous breakpoint optimization in smooth local-support curve fitting. Appl Math Comput 275:195-212

Gálvez A, Iglesias A, Cobo A, Puig-Pey J, Espinola J (2007) Bézier curve and surface fitting of $3 \mathrm{D}$ point clouds through genetic algorithms, functional networks and least-squares approximation.In: Lectures notes in computer science, vol 4706, pp 680-693

Gálvez A, Cobo A, Puig-Pey J, Iglesias A (2008) Particle swarm optimization for Bézier surface reconstruction. In: Lectures notes in computer science, vol 5102, pp 116-125

Gálvez A, Iglesias A, Puig-Pey J (2012) Iterative two-step geneticalgorithm method for efficient polynomial B-spline surface reconstruction. Inf Sci 182(1):56-76

Gálvez A, Iglesias A, Avila A (2013) Immunological-based approach for accurate fitting of 3D noisy data points with Bézier surfaces. In: Proceedings of International Conference on Computer Science-ICCS'2013, Procedia Computer Science vol 18, pp 50-59

Gálvez A, Iglesias A, Avila A, Otero C, Arias R, Manchado C (2015) Elitist clonal selection algorithm for optimal choice of free knots in B-spline data fitting. Appl Soft Comput 26:90-106

Gordon WJ (1969) Spline-blended surface interpolation through curve networks. J Math Mech 18(10):931-952

Gu P, Yan X (1995) Neural network approach to the reconstruction of free-form surfaces for reverse engineering. Comput Aided Des 27(1):59-64

Hoffmann M (2005) Numerical control of Kohonen neural network for scattered data approximation. Numer Algorithms 39:175-186

Iglesias A, Echevarría G, Gálvez A (2004) Functional networks for B-spline surface reconstruction. Future Gener Comput Syst 20(8):1337-1353

Iglesias A, Gálvez A (2014) Hybrid functional-neural approach for surface reconstruction. Math Problems Eng, Article ID 351648

Iglesias A, Gálvez A (2015) Memetic electromagnetism algorithm for finite approximation with rational Bézier curves. In: Tan Y, Shi Y, Buarque F, Gelbukh A, Das S, Engelbrecht A (eds) Adv Swarm Comput Intell. Springer, Berlin, pp 30-40

Iglesias A, Gálvez A (2001) Applying functional networks to fit data points from B-spline surfaces. In: Proceedings of Computer Graphics International, CGI'2001, Hong-Kong (China). IEEE Computer Society Press, Los Alamitos, CA, pp 329-332

Jing L, Sun L (2005) Fitting B-spline curves by least squares support vector machines. In: Proceedings of the second international conference on neural networks and brain. Beijing (China). IEEE Press, New York, pp 905-909

Jones M, Chen M (1994) A new approach to the construction of surfaces from contour data. Comput Graph Forum 13(3):75-84 
Leu MC, Peng X, Zhang W (2005) Surface reconstruction for interactive modeling of free-form solids by virtual sculpting. CIRP Ann Manuf Technol 54(1):131-134

Levoy M, Pulli K, Curless B, Rusinkiewicz S, Koller D, Pereira L, Ginzton M, Anderson S, Davis J, Ginsberg J, Shade J, Fulk D (2000) The digital michelangelo project: 3D scanning of large statues. In: SIGGRAPH 2000, New Orleans, pp 131-144

Li W, Xu S, Zhao G, Goh LP (2005) Adaptive knot placement in B-spline curve approximation. Comput Aided Des 37:791-797

Lim C, Turkiyyah G, Ganter M, Storti D (1995) Implicit reconstruction of solids from cloud point sets. In: Proceedings of 1995 ACM symposium on solid modeling, Salt Lake City, Utah, pp 393-402

Luus R, Jaakola THI (1973) Optimization by direct search and systematic reduction of the size of search region. Am Inst Chem Eng J 19(4):760-766

Ma WY, Kruth JP (1995) Parameterization of randomly measured points for least squares fitting of B-spline curves and surfaces. Comput Aided Des 27(9):663-675

Maekawa I, Ko K (2002) Surface construction by fitting unorganized curves. Graph Models 64:316-332

Meyers D, Skinnwer S, Sloan K (1992) Surfaces from contours. ACM Trans Graph 11(3):228-258

Park H (2004) An error-bounded approximate method for representing planar curves in B-splines. Comput Aided Geom Des 21:479-497

Park H, Kim K (1997) Smooth surface approximation to serial crosssections. Comput Aided Des 28(12):995-1005

Park H, Lee JH (2007) B-spline curve fitting based on adaptive curve refinement using dominant points. Comput Aided Des 39:439-451

Patrikalakis NM, Maekawa T (2002) Shape interrogation for computer aided design and manufacturing. Springer, Heidelberg

Piegl L, Tiller W (1997) The NURBS book. Springer, Berlin
Pottmann H, Leopoldseder S, Hofer M (2002) Approximation with active b-spline curves and surfaces. In: Proceedings of pacific graphics. IEEE Computer Society Press, pp 8-25

Pottmann H, Leopoldseder S, Hofer M, Steiner T, Wang W (2005) Industrial geometry: recent advances and applications in CAD. Comput Aided Des 37:751-766

Prasad M, Zisserman A, Fitzgibbon AW (2006) Single view reconstruction of curved surfaces. In: Proceedings of the IEEE conference on computer vision and pattern recognition, vol 2. IEEE Computer Society Press, Los Alamitos, CA, pp 1345-1354

Pratt V (1987) Direct least-squares fitting of algebraic surfaces. Proc SIGGRAPH' 87 Comput Graph 21(4):145-152

Sarfraz M, Raza SA (2001) Capturing outline of fonts using genetic algorithms and splines. In: Proceedings of fifth international conference on information visualization IV'2001, IEEE Computer Society Press, pp 738-743

Savchenko V, Pasko A, Okunev O, Kunii T (1995) Function representation of solids reconstructed from scattered surface points and contours. Comput Graph Forum 14(4):181-188

Schmitt F, Barsky BA, Du W (1986) An adaptive subdivision method for surface fitting from sampled data. Proc SIGGRAPH'86 Comput Graph 20(4):179-188

Sclaroff S, Pentland A (1991) Generalized implicit functions for computer graphics. Proc SIGGRAPH'91 Comput Graph 25(4):247-250

Varady T, Martin R (2002) Reverse engineering. In: Farin G, Hoschek J, Kim M (eds) Handbook of computer aided geometric design. Elsevier Science, Amsterdam

Yoshimoto F, Harada T, Yoshimoto Y (2003) Data fitting with a spline using a real-coded algorithm. Comput Aided Des $35: 751-760$

Zhao X, Zhang C, Yang B, Li P (2011) Adaptive knot adjustment using a GMM-based continuous optimization algorithm in B-spline curve approximation. Comput Aided Des 43:598-604 